\title{
Real Vector Space and Related Notions 1
}

\author{
Kazuhisa Nakasho \\ Yamaguchi University \\ Yamaguchi, Japan
}

\author{
Hiroyuki Okazaki \\ Shinshu University \\ Nagano, Japan
}

\author{
Yasunari Shidama \\ Shinshu University \\ Nagano, Japan
}

\begin{abstract}
Summary. In this paper, we discuss the properties that hold in finite dimensional vector spaces and related spaces. In the Mizar language [1, 2], variables are strictly typed, and their type conversion requires a complicated process. Our purpose is to formalize that some properties of finite dimensional vector spaces are preserved in type transformations, and to contain the complexity of type transformations into this paper. Specifically, we show that properties such as algebraic structure, subsets, finite sequences and their sums, linear combination, linear independence, and affine independence are preserved in type conversions among TOP-REAL (n), REAL-NS (n), and n-VectSp_over F_Real. We referred to [4, [9], and [8] in the formalization.
\end{abstract}

MSC: 15A03 46B15 68V20

Keywords: real vector space; topological space; normed spaces

MML identifier: REAL_NS2, version: 8.1.11 5.66.1402

\section{Common Properties Between Norm and Topology in Finite Dimensional Linear Spaces}

From now on $X$ denotes a set, $n, m, k$ denote natural numbers, $K$ denotes a field, $f$ denotes an $n$-element, real-valued finite sequence, and $M$ denotes a matrix over $\mathbb{R}_{\mathrm{F}}$ of dimension $n \times m$.

Now we state the propositions:

\footnotetext{
${ }^{1}$ This study was supported in part by JSPS KAKENHI Grant Numbers 17K00182 and 20K19863. 
(1) The RLS structure of $\mathcal{E}_{\mathrm{T}}^{n}=$ the RLS structure of $\left\langle\mathcal{E}^{n},\|\cdot\|\right\rangle$.

Proof: For every elements $x, y$ of $\mathcal{R}^{n},+_{\mathcal{E}^{n}}(x, y)=+_{\mathbb{R}^{\operatorname{Seg} n}}(x, y)$. For every element $x$ of $\mathbb{R}$ and for every element $y$ of $\mathcal{R}^{n}, \cdot \mathcal{E}^{n}(x, y)=\cdot \mathbb{R}_{\mathbb{R}^{\operatorname{Seg} n}}(x, y)$ by [3, (3)].

(2) $\mathcal{E}^{n}=\operatorname{MetricSpaceNorm}\left\langle\mathcal{E}^{n},\|\cdot\|\right\rangle$.

Proof: Set $X=\left\langle\mathcal{E}^{n},\|\cdot\|\right\rangle$. For every elements $x, y$ of $\mathcal{R}^{n}$, (the distance of $\left.\mathcal{E}^{n}\right)(x, y)=($ the distance by norm of $X)(x, y)$.

(3) The topological structure of $\mathcal{E}_{\mathrm{T}}^{n}=\operatorname{TopSpaceNorm}\left\langle\mathcal{E}^{n},\|\cdot\|\right\rangle$. The theorem is a consequence of (2).

(4) The carrier of $\mathcal{E}_{\mathrm{T}}^{n}=$ the carrier of $\left\langle\mathcal{E}^{n},\|\cdot\|\right\rangle$. The theorem is a consequence of $(1)$.

(5) The carrier of the $n$-dimension vector space over $\mathbb{R}_{\mathrm{F}}=$ the carrier of $\left\langle\mathcal{E}^{n},\|\cdot\|\right\rangle$. The theorem is a consequence of (4).

(6) $0_{\mathcal{E}_{\mathrm{T}}^{n}}=0_{\left\langle\mathcal{E}^{n},\|\cdot\|\right\rangle}$. The theorem is a consequence of (1).

(7) Let us consider elements $p, q$ of $\mathcal{E}_{\mathrm{T}}^{n}$, and elements $f, g$ of $\left\langle\mathcal{E}^{n},\|\cdot\|\right\rangle$. If $p=f$ and $q=g$, then $p+q=f+g$. The theorem is a consequence of (1).

(8) Let us consider a real number $r$, an element $q$ of $\mathcal{E}_{\mathrm{T}}^{n}$, and an element $g$ of $\left\langle\mathcal{E}^{n},\|\cdot\|\right\rangle$. If $q=g$, then $r \cdot q=r \cdot g$. The theorem is a consequence of (1).

(9) Let us consider an element $q$ of $\mathcal{E}_{\mathrm{T}}^{n}$, and an element $g$ of $\left\langle\mathcal{E}^{n},\|\cdot\|\right\rangle$. If $q=g$, then $-q=-g$. The theorem is a consequence of (8).

(10) Let us consider elements $p, q$ of $\mathcal{E}_{\mathrm{T}}^{n}$, and elements $f, g$ of $\left\langle\mathcal{E}^{n},\|\cdot\|\right\rangle$. If $p=f$ and $q=g$, then $p-q=f-g$. The theorem is a consequence of (9) and $(7)$.

Let us consider a set $X$ and a natural number $n$.

(11) $X$ is a linear combination of $\left\langle\mathcal{E}^{n},\|\cdot\|\right\rangle$ if and only if $X$ is a linear combination of $\mathcal{E}_{\mathrm{T}}^{n}$. The theorem is a consequence of (4).

(12) $X$ is a linear combination of $\left\langle\mathcal{E}^{n},\|\cdot\|\right\rangle$ if and only if $X$ is a linear combination of the $n$-dimension vector space over $\mathbb{R}_{F}$. The theorem is a consequence of (11).

(13) Let us consider a linear combination $L_{5}$ of $\mathcal{E}_{\mathrm{T}}^{n}$, and a linear combination $L_{2}$ of $\left\langle\mathcal{E}^{n},\|\cdot\|\right\rangle$. Suppose $L_{2}=L_{5}$. Then the support of $L_{2}=$ the support of $L_{5}$.

(14) Let us consider a linear combination $L_{5}$ of the $n$-dimension vector space over $\mathbb{R}_{\mathrm{F}}$, and a linear combination $L_{2}$ of $\left\langle\mathcal{E}^{n},\|\cdot\|\right\rangle$. Suppose $L_{2}=L_{5}$. Then the support of $L_{2}=$ the support of $L_{5}$. The theorem is a consequence of (11).

Let us consider a set $F$. Now we state the propositions: 
(15) $F$ is a subset of $\mathcal{E}_{\mathrm{T}}^{n}$ if and only if $F$ is a subset of $\left\langle\mathcal{E}^{n},\|\cdot\|\right\rangle$.

(16) $F$ is a subset of $\left\langle\mathcal{E}^{n},\|\cdot\|\right\rangle$ if and only if $F$ is a subset of the $n$-dimension vector space over $\mathbb{R}_{\mathrm{F}}$.

(17) $F$ is a finite sequence of elements of $\mathcal{E}_{\mathrm{T}}^{n}$ if and only if $F$ is a finite sequence of elements of $\left\langle\mathcal{E}^{n},\|\cdot\|\right\rangle$.

(18) $F$ is a function from $\mathcal{E}_{\mathrm{T}}^{n}$ into $\mathbb{R}$ if and only if $F$ is a function from $\left\langle\mathcal{E}^{n},\|\cdot\|\right\rangle$ into $\mathbb{R}$. The theorem is a consequence of (4).

(19) Let us consider a finite sequence $F_{2}$ of elements of $\mathcal{E}_{\mathrm{T}}^{n}$, a function $f_{1}$ from $\mathcal{E}_{\mathrm{T}}^{n}$ into $\mathbb{R}$, a finite sequence $F_{4}$ of elements of $\left\langle\mathcal{E}^{n},\|\cdot\|\right\rangle$, and a function $f_{3}$ from $\left\langle\mathcal{E}^{n},\|\cdot\|\right\rangle$ into $\mathbb{R}$. If $f_{1}=f_{3}$ and $F_{2}=F_{4}$, then $f_{1} \cdot F_{2}=f_{3} \cdot F_{4}$. The theorem is a consequence of (4) and (8).

(20) Let us consider a finite sequence $F$ of elements of $\left\langle\mathcal{E}^{n},\|\cdot\|\right\rangle$, a function $f_{1}$ from $\left\langle\mathcal{E}^{n},\|\cdot\|\right\rangle$ into $\mathbb{R}$, a finite sequence $F_{4}$ of elements of the $n$-dimension vector space over $\mathbb{R}_{\mathrm{F}}$, and a function $f_{3}$ from the $n$-dimension vector space over $\mathbb{R}_{\mathrm{F}}$ into $\mathbb{R}_{\mathrm{F}}$. If $f_{1}=f_{3}$ and $F=F_{4}$, then $f_{1} \cdot F=f_{3} \cdot F_{4}$. The theorem is a consequence of (18), (4), and (19).

(21) Let us consider a finite sequence $F_{3}$ of elements of $\mathcal{E}_{\mathrm{T}}^{n}$, and a finite sequence $F_{2}$ of elements of $\left\langle\mathcal{E}^{n},\|\cdot\|\right\rangle$. If $F_{3}=F_{2}$, then $\sum F_{3}=\sum F_{2}$.

Proof: Set $T=\mathcal{E}_{\mathrm{T}}^{n}$. Set $V=\left\langle\mathcal{E}^{n},\|\cdot\|\right\rangle$. Consider $f$ being a sequence of the carrier of $T$ such that $\sum F=f(\operatorname{len} F)$ and $f(0)=0_{T}$ and for every natural number $j$ and for every element $v$ of $T$ such that $j<\operatorname{len} F$ and $v=F(j+1)$ holds $f(j+1)=f(j)+v$.

Consider $f_{3}$ being a sequence of the carrier of $V$ such that $\sum F_{4}=$ $f_{3}\left(\right.$ len $\left.F_{4}\right)$ and $f_{3}(0)=0_{V}$ and for every natural number $j$ and for every element $v$ of $V$ such that $j<\operatorname{len} F_{4}$ and $v=F_{4}(j+1)$ holds $f_{3}(j+1)=$ $f_{3}(j)+v$. Define $\mathcal{S}$ [natural number] $\equiv$ if $\$_{1} \leqslant \operatorname{len} F$, then $f\left(\$_{1}\right)=f_{3}\left(\$_{1}\right)$. For every natural number $i$ such that $\mathcal{S}[i]$ holds $\mathcal{S}[i+1]$. $\mathcal{S}[0]$. For every natural number $n, \mathcal{S}[n]$.

(22) Let us consider a finite sequence $F$ of elements of $\left\langle\mathcal{E}^{n},\|\cdot\|\right\rangle$, and a finite sequence $F_{4}$ of elements of the $n$-dimension vector space over $\mathbb{R}_{\mathrm{F}}$. If $F_{4}=$ $F$, then $\sum F=\sum F_{4}$. The theorem is a consequence of (4) and (21).

(23) Let us consider a linear combination $L_{2}$ of $\left\langle\mathcal{E}^{n},\|\cdot\|\right\rangle$, and a linear combination $L_{4}$ of $\mathcal{E}_{\mathrm{T}}^{n}$. If $L_{2}=L_{4}$, then $\sum L_{2}=\sum L_{4}$. The theorem is a consequence of (4), (19), and (21).

(24) Let us consider a linear combination $L_{5}$ of the $n$-dimension vector space over $\mathbb{R}_{\mathrm{F}}$, and a linear combination $L_{2}$ of $\left\langle\mathcal{E}^{n},\|\cdot\|\right\rangle$. If $L_{2}=L_{5}$, then $\sum L_{2}=\sum L_{5}$. The theorem is a consequence of (11) and (23).

(25) Let us consider a subset $A_{3}$ of $\left\langle\mathcal{E}^{n},\|\cdot\|\right\rangle$, and a subset $A_{4}$ of $\mathcal{E}_{\mathrm{T}}^{n}$. Suppose $A_{3}=A_{4}$. Let us consider an object $X$. Then $X$ is a linear combination 
of $A_{3}$ if and only if $X$ is a linear combination of $A_{4}$. The theorem is a consequence of (11).

(26) Let us consider a subset $A_{3}$ of $\left\langle\mathcal{E}^{n},\|\cdot\|\right\rangle$, and a subset $A_{4}$ of $\mathcal{E}_{\mathrm{T}}^{n}$. If $A_{3}=A_{4}$, then $\Omega_{\operatorname{Lin}\left(A_{3}\right)}=\Omega_{\operatorname{Lin}\left(A_{4}\right)}$. The theorem is a consequence of (11) and (23).

(27) Let us consider a subset $A_{2}$ of the $n$-dimension vector space over $\mathbb{R}_{\mathrm{F}}$, and a subset $A_{3}$ of $\left\langle\mathcal{E}^{n},\|\cdot\|\right\rangle$. If $A_{2}=A_{3}$, then $\Omega_{\operatorname{Lin}\left(A_{3}\right)}=\Omega_{\operatorname{Lin}\left(A_{2}\right)}$. The theorem is a consequence of (4) and (26).

(28) Let us consider a subset $A_{3}$ of $\left\langle\mathcal{E}^{n},\|\cdot\|\right\rangle$, and a subset $A_{4}$ of $\mathcal{E}_{\mathrm{T}}^{n}$. Suppose $A_{3}=A_{4}$. Then $A_{3}$ is linearly independent if and only if $A_{4}$ is linearly independent. The theorem is a consequence of (11), (6), and (23).

(29) Let us consider a subset $A_{2}$ of the $n$-dimension vector space over $\mathbb{R}_{\mathrm{F}}$, and a subset $A_{3}$ of $\left\langle\mathcal{E}^{n},\|\cdot\|\right\rangle$. Suppose $A_{2}=A_{3}$. Then $A_{2}$ is linearly independent if and only if $A_{3}$ is linearly independent. The theorem is a consequence of (4) and (28).

(30) Let us consider an object $X$. Then $X$ is a subspace of $\left\langle\mathcal{E}^{n},\|\cdot\|\right\rangle$ if and only if $X$ is a subspace of $\mathcal{E}_{\mathrm{T}}^{n}$. The theorem is a consequence of (1), (4), and (6).

(31) Let us consider a set $X$, a subspace $U$ of $\left\langle\mathcal{E}^{n},\|\cdot\|\right\rangle$, and a subspace $W$ of the $n$-dimension vector space over $\mathbb{R}_{\mathrm{F}}$. Suppose $\Omega_{U}=\Omega_{W}$. Then $X$ is a linear combination of $U$ if and only if $X$ is a linear combination of $W$. The theorem is a consequence of (30).

(32) Let us consider a one-to-one finite sequence $F$ of elements of $\left\langle\mathcal{E}^{n},\|\cdot\|\right\rangle$. Suppose $\operatorname{rng} F$ is linearly independent. Then there exists a square matrix $M$ over $\mathbb{R}_{\mathrm{F}}$ of dimension $n$ such that

(i) $M$ is invertible, and

(ii) $M \uparrow$ len $F=F$.

The theorem is a consequence of (4) and (28).

(33) Let us consider a square matrix $M$ over $\mathbb{R}_{\mathrm{F}}$ of dimension $n$, and a square matrix $N$ over $\mathbb{R}$ of dimension $n$. Suppose $N=\left(\mathbb{R}_{\mathrm{F}} \rightarrow \mathbb{R}\right) M$. Then $M$ is invertible if and only if $N$ is invertible.

(34) Let us consider a square matrix $M$ over $\mathbb{R}$ of dimension $n$. Then $M$ is invertible if and only if $\left(\mathbb{R} \rightarrow \mathbb{R}_{\mathrm{F}}\right.$ ) $M$ is invertible.

(35) Let us consider a one-to-one finite sequence $F$ of elements of $\left\langle\mathcal{E}^{n},\|\cdot\|\right\rangle$. Suppose $\operatorname{rng} F$ is linearly independent. Then there exists a square matrix $M$ over $\mathbb{R}$ of dimension $n$ such that

(i) $M$ is invertible, and 
(ii) $M \uparrow \operatorname{len} F=F$.

The theorem is a consequence of (32) and (33).

(36) Let us consider a one-to-one finite sequence $F$ of elements of $\left\langle\mathcal{E}^{n},\|\cdot\|\right\rangle$. Suppose $\operatorname{rng} F$ is linearly independent. Let us consider an ordered basis $B$ of the $n$-dimension vector space over $\mathbb{R}_{\mathrm{F}}$. Suppose $B=\operatorname{MX} 2$ FinS $I_{\mathbb{R}_{\mathrm{F}}}^{n \times n}$. Let us consider a square matrix $M$ over $\mathbb{R}_{\mathrm{F}}$ of dimension $n$. Suppose $M$ is invertible and $M \uparrow \operatorname{len} F=F$. Then $(\operatorname{Mx} 2 \operatorname{Tran}(M))^{\circ}\left(\Omega_{\operatorname{Lin}(\operatorname{rng}(B \nmid \operatorname{len} F))}\right)=$ $\Omega_{\operatorname{Lin}(\operatorname{rng} F)}$. The theorem is a consequence of (4), (28), and (26).

(37) Let us consider linearly independent subsets $A, B$ of $\left\langle\mathcal{E}^{n},\|\cdot\|\right\rangle$. Suppose $\overline{\bar{A}}=\overline{\bar{B}}$. Then there exists a square matrix $M$ over $\mathbb{R}_{\mathrm{F}}$ of dimension $n$ such that

(i) $M$ is invertible, and

(ii) $(\operatorname{Mx} 2 \operatorname{Tran}(M))^{\circ}\left(\Omega_{\operatorname{Lin}(A)}\right)=\Omega_{\operatorname{Lin}(B)}$.

The theorem is a consequence of (4), (28), and (26).

(38) Let us consider natural numbers $n, m$, a matrix $M$ over $\mathbb{R}_{\mathrm{F}}$ of dimension $n \times m$, and a linearly independent subset $A$ of $\left\langle\mathcal{E}^{n},\|\cdot\|\right\rangle$. Suppose $\operatorname{rk}(M)=n$. Then $(\operatorname{Mx} 2 \operatorname{Tran}(M))^{\circ} A$ is linearly independent. The theorem is a consequence of (4) and (28).

(39) Let us consider an element $p$ of $\mathcal{E}_{\mathrm{T}}^{n}$, an element $f$ of $\left\langle\mathcal{E}^{n},\|\cdot\|\right\rangle$, a subset $H$ of $\mathcal{E}_{\mathrm{T}}^{n}$, and a subset $I$ of $\left\langle\mathcal{E}^{n},\|\cdot\|\right\rangle$. If $p=f$ and $H=I$, then $p+H=f+I$. The theorem is a consequence of (4) and (7).

(40) Let us consider a subset $A_{3}$ of $\left\langle\mathcal{E}^{n},\|\cdot\|\right\rangle$, and a subset $A_{4}$ of $\mathcal{E}_{\mathrm{T}}^{n}$. If $A_{3}=A_{4}$, then $A_{3}$ is affine iff $A_{4}$ is affine. The theorem is a consequence of (4), (8), and (7).

(41) Let us consider a set $X$. Then $X$ is an affinely independent subset of $\left\langle\mathcal{E}^{n},\|\cdot\|\right\rangle$ if and only if $X$ is an affinely independent subset of $\mathcal{E}_{\mathrm{T}}^{n}$. The theorem is a consequence of (4), (6), (9), (39), and (28).

(42) Let us consider natural numbers $n, m$, a matrix $M$ over $\mathbb{R}_{\mathrm{F}}$ of dimension $n \times m$, and an affinely independent subset $A$ of $\left\langle\mathcal{E}^{n},\|\cdot\|\right\rangle$. Suppose $\operatorname{rk}(M)=n$. Then $(\operatorname{Mx} 2 \operatorname{Tran}(M))^{\circ} A$ is affinely independent. The theorem is a consequence of (41).

(43) Let us consider a subset $A_{3}$ of $\left\langle\mathcal{E}^{n},\|\cdot\|\right\rangle$, and a subset $A_{4}$ of $\mathcal{E}_{\mathrm{T}}^{n}$. If $A_{3}=A_{4}$, then Affin $A_{3}=$ Affin $A_{4}$. The theorem is a consequence of (4) and (40).

(44) Let us consider a linear combination $L$ of $\left\langle\mathcal{E}^{n},\|\cdot\|\right\rangle$, and a linear combination $S$ of $\mathcal{E}_{\mathrm{T}}^{n}$. If $L=S$, then $\operatorname{sum} L=\operatorname{sum} S$. The theorem is a consequence of $(4)$. 
(45) Let us consider a subset $A_{3}$ of $\left\langle\mathcal{E}^{n},\|\cdot\|\right\rangle$, a subset $A_{4}$ of $\mathcal{E}_{\mathrm{T}}^{n}$, an element $v$ of $\left\langle\mathcal{E}^{n},\|\cdot\|\right\rangle$, and an element $w$ of $\mathcal{E}_{\mathrm{T}}^{n}$. Suppose $A_{3}=A_{4}$ and $v=w$ and $v \in$ Affin $A_{3}$ and $A_{3}$ is affinely independent. Then $v \rightarrow A_{3}=w \rightarrow A_{4}$. The theorem is a consequence of (41), (25), (23), (44), and (43).

(46) Let us consider natural numbers $n, m$, a matrix $M$ over $\mathbb{R}_{\mathrm{F}}$ of dimension $n \times m$, and an affinely independent subset $A$ of $\left\langle\mathcal{E}^{n},\|\cdot\|\right\rangle$. Suppose $\operatorname{rk}(M)=$ $n$. Let us consider an element $v$ of $\left\langle\mathcal{E}^{n},\|\cdot\|\right\rangle$. Suppose $v \in$ Affin $A$. Then

(i) $(\operatorname{Mx} 2 \operatorname{Tran}(M))(v) \in \operatorname{Affin}\left((\operatorname{Mx} 2 \operatorname{Tran}(M))^{\circ} A\right)$, and

(ii) for every $n$-element, real-valued finite sequence $f,(v \rightarrow A)(f)=$ $\left((\operatorname{Mx} 2 \operatorname{Tran}(M))(v) \rightarrow(\operatorname{Mx} 2 \operatorname{Tran}(M))^{\circ} A\right)((\operatorname{Mx} 2 \operatorname{Tran}(M))(f))$.

The theorem is a consequence of (41), (4), (43), and (45).

(47) Let us consider natural numbers $n, m$, a matrix $M$ over $\mathbb{R}_{F}$ of dimension $n \times m$, and a linearly independent subset $A$ of $\left\langle\mathcal{E}^{m},\|\cdot\|\right\rangle$. Suppose $\operatorname{rk}(M)=n$. Then $(\operatorname{Mx} 2 \operatorname{Tran}(M))^{-1}(A)$ is linearly independent. The theorem is a consequence of (4) and (28).

(48) Let us consider natural numbers $n, m$, a matrix $M$ over $\mathbb{R}_{\mathrm{F}}$ of dimension $n \times m$, and an affinely independent subset $A$ of $\left\langle\mathcal{E}^{m},\|\cdot\|\right\rangle$. Suppose $\operatorname{rk}(M)=n$. Then $(\mathrm{Mx} 2 \operatorname{Tran}(M))^{-1}(A)$ is affinely independent. The theorem is a consequence of (41).

(49) Let us consider a real linear space $V$. Then every strict subspace of $V$ is a strict subspace of $\Omega_{V}$.

(50) Let us consider a set $X$. Then $X$ is a basis of the $n$-dimension vector space over $\mathbb{R}_{\mathrm{F}}$ if and only if $X$ is a basis of $\mathcal{E}_{\mathrm{T}}^{n}$.

Let us consider a non empty natural number $n$.

(51) $+_{\mathbb{R}^{\operatorname{Seg} n}}=\pi^{n}$ (the addition of $\mathbb{R}_{\mathrm{F}}$ ).

ProOF: Set $O_{1}=+_{\mathbb{R}^{S e g} n}$. Set $O_{2}=\pi^{n}$ (the addition of $\left.\mathbb{R}_{F}\right)$. For every elements $x, y$ of $\mathcal{R}^{n}, O_{1}(x, y)=O_{2}(x, y)$.

(52) $\cdot \cdot_{\mathbb{R}^{\operatorname{Seg} n}}^{\mathbb{R}}=\cdot_{\mathbb{R}_{\mathrm{F}}}^{n}$.

PROOF: Set $O_{1}=\cdot_{\mathbb{R}^{\operatorname{Seg} n}}$. Set $O_{2}=\cdot_{\mathbb{R}_{\mathrm{F}}}^{n}$. For every element $x$ of $\mathbb{R}$ and for every element $y$ of $\mathcal{R}^{n}, O_{1}(x, y)=O_{2}(x, y)$.

(53) (i) $\mathcal{E}_{\mathrm{T}}^{n}$ is finite dimensional, and

(ii) $\operatorname{dim}\left(\mathcal{E}_{\mathrm{T}}^{n}\right)=n$.

The theorem is a consequence of (50).

(54) Let us consider a non empty natural number $n$. Then

(i) the carrier of $\mathcal{E}_{\mathrm{T}}^{n}=$ the carrier of the $n$-dimension vector space over $\mathbb{R}_{\mathrm{F}}$, and

(ii) $0_{\mathcal{E}_{\mathrm{T}}^{n}}=0_{\alpha}$, and 
(iii) the addition of $\mathcal{E}_{\mathrm{T}}^{n}=$ the addition of the $n$-dimension vector space over $\mathbb{R}_{F}$, and

(iv) the external multiplication of $\mathcal{E}_{\mathrm{T}}^{n}=$ the left multiplication of the $n$-dimension vector space over $\mathbb{R}_{\mathrm{F}}$,

where $\alpha$ is the $n$-dimension vector space over $\mathbb{R}_{F}$. The theorem is a consequence of (51) and (52).

(55) Let us consider a non empty natural number $n$, elements $x_{2}, y_{2}$ of the $n$ dimension vector space over $\mathbb{R}_{\mathrm{F}}$, and elements $x_{1}, y_{1}$ of $\mathcal{E}_{\mathrm{T}}^{n}$. If $x_{2}=x_{1}$ and $y_{2}=y_{1}$, then $x_{2}+y_{2}=x_{1}+y_{1}$.

(56) Let us consider a non empty natural number $n$, an element $a_{1}$ of $\mathbb{R}_{\mathrm{F}}$, a real number $a_{2}$, an element $x_{2}$ of the $n$-dimension vector space over $\mathbb{R}_{\mathrm{F}}$, and an element $x_{1}$ of $\mathcal{E}_{\mathrm{T}}^{n}$. If $a_{1}=a_{2}$ and $x_{2}=x_{1}$, then $a_{1} \cdot x_{2}=a_{2} \cdot x_{1}$.

(57) Let us consider a non empty natural number $n$, an element $x_{2}$ of the $n$ dimension vector space over $\mathbb{R}_{\mathrm{F}}$, and an element $x_{1}$ of $\mathcal{E}_{\mathrm{T}}^{n}$. If $x_{2}=x_{1}$, then $-x_{2}=-x_{1}$. The theorem is a consequence of (54).

(58) Let us consider a non empty natural number $n$, elements $x_{2}, y_{2}$ of the $n$ dimension vector space over $\mathbb{R}_{\mathrm{F}}$, and elements $x_{1}, y_{1}$ of $\mathcal{E}_{\mathrm{T}}^{n}$. If $x_{2}=x_{1}$ and $y_{2}=y_{1}$, then $x_{2}-y_{2}=x_{1}-y_{1}$. The theorem is a consequence of (57) and (54).

(59) Let us consider a non empty natural number $n$, a subset $A_{4}$ of $\mathcal{E}_{\mathrm{T}}^{n}$, and a subset $A_{3}$ of the $n$-dimension vector space over $\mathbb{R}_{\mathrm{F}}$. Suppose $A_{4}=A_{3}$. Then

(i) the carrier of $\operatorname{Lin}\left(A_{4}\right)=$ the carrier of $\operatorname{Lin}\left(A_{3}\right)$, and

(ii) $0_{\operatorname{Lin}\left(A_{4}\right)}=0_{\operatorname{Lin}\left(A_{3}\right)}$, and

(iii) the addition of $\operatorname{Lin}\left(A_{4}\right)=$ the addition of $\operatorname{Lin}\left(A_{3}\right)$, and

(iv) the external multiplication of $\operatorname{Lin}\left(A_{4}\right)=$ the left multiplication of $\operatorname{Lin}\left(A_{3}\right)$.

The theorem is a consequence of (54).

(60) Let us consider a subset $A_{4}$ of $\mathcal{E}_{\mathrm{T}}^{n}$, and a subset $A_{3}$ of $\left\langle\mathcal{E}^{n},\|\cdot\|\right\rangle$. If $A_{4}=A_{3}$, then $\operatorname{Lin}\left(A_{4}\right)=\operatorname{Lin}\left(A_{3}\right)$. The theorem is a consequence of (26) and (1).

(61) Let us consider a set $X$. Then $X$ is a basis of $\mathcal{E}_{\mathrm{T}}^{n}$ if and only if $X$ is a basis of $\left\langle\mathcal{E}^{n},\|\cdot\|\right\rangle$. The theorem is a consequence of (4), (28), (49), and (26).

(62) (i) $\left\langle\mathcal{E}^{n},\|\cdot\|\right\rangle$ is finite dimensional, and

(ii) $\operatorname{dim}\left(\left\langle\mathcal{E}^{n},\|\cdot\|\right\rangle\right)=n$.

The theorem is a consequence of (53), (4), and (61). 


\section{Finite Dimensional Vector Spaces over Real Field}

Note that there exists a real normed space which is finite dimensional. Now we state the propositions:

(63) Let us consider a field $K$, a finite dimensional vector space $V$ over $K$, and an ordered basis $b$ of $V$. Then there exists a linear transformation $T$ from $V$ to the $\operatorname{dim}(V)$-dimension vector space over $K$ such that

(i) $T$ is bijective, and

(ii) for every element $x$ of $V, T(x)=x \rightarrow b$.

Proof: Set $W=$ the $\operatorname{dim}(V)$-dimension vector space over $K$. Define $\mathcal{P}$ [object, object] $\equiv$ there exists an element $x$ of $V$ such that $\$_{1}=x$ and $\$_{2}=x \rightarrow b$.

For every element $x$ of the carrier of $V$, there exists an element $y$ of the carrier of $W$ such that $\mathcal{P}[x, y]$. Consider $f$ being a function from the carrier of $V$ into the carrier of $W$ such that for every element $x$ of the carrier of $V, \mathcal{P}[x, f(x)]$. For every element $x$ of $V, f(x)=x \rightarrow b$. For every elements $x, y$ of $V, f(x+y)=f(x)+f(y)$. For every scalar $a$ of $K$ and for every vector $x$ of $V, f(a \cdot x)=a \cdot f(x)$. For every objects $x, y$ such that $x, y \in \operatorname{dom} f$ and $f(x)=f(y)$ holds $x=y$.

For every object $y$ such that $y \in$ the carrier of $W$ there exists an object $x$ such that $x \in$ the carrier of $V$ and $y=f(x)$ by [6, (102)], [7, (21)], [5, $(36)]$.

(64) Let us consider a field $K$, and a finite dimensional vector space $V$ over $K$. Then there exists a linear transformation $T$ from $V$ to the $\operatorname{dim}(V)$ dimension vector space over $K$ such that $T$ is bijective. The theorem is a consequence of (63).

(65) Let us consider a field $K$, and finite dimensional vector spaces $V, W$ over $K$. Then $\operatorname{dim}(V)=\operatorname{dim}(W)$ if and only if there exists a linear transformation $T$ from $V$ to $W$ such that $T$ is bijective. The theorem is a consequence of (64).

(66) Let us consider a real linear space $X$. Then

(i) the carrier of $X=$ the carrier of $\operatorname{RLSp2RVSp}(X)$, and

(ii) the zero of $X=$ the zero of $\operatorname{RLSp} 2 \operatorname{RVSp}(X)$, and

(iii) the addition of $X=$ the addition of $\operatorname{RLSp} 2 \operatorname{RVSp}(X)$, and

(iv) the external multiplication of $X=$ the left multiplication of $\operatorname{RLSp} 2 \operatorname{RVSp}(X)$.

(67) Let us consider a strict real linear space $X$. Then RVSp2RLSp RLSp2RVSp $(X)=X$. 
(68) Let us consider a strict vector space $X$ over $\mathbb{R}_{\mathrm{F}}$.

Then RLSp2RVSp(RVSp2RLSp $X)=X$.

Let us consider a real linear space $V$ and a set $F$.

(69) $F$ is a subset of $V$ if and only if $F$ is a subset of $\operatorname{RLSp} 2 \operatorname{RVSp}(V)$.

(70) $F$ is a finite sequence of elements of $V$ if and only if $F$ is a finite sequence of elements of RLSp2RVSp $(V)$.

(71) $F$ is a function from $V$ into $\mathbb{R}$ if and only if $F$ is a function from $\operatorname{RLSp} 2 \operatorname{RVSp}(V)$ into $\mathbb{R}$.

(72) Let us consider a real linear space $T$, and a set $X$. Then $X$ is a linear combination of $\operatorname{RLSp} 2 \operatorname{RVSp}(T)$ if and only if $X$ is a linear combination of $T$.

(73) Let us consider a real linear space $T$, a linear combination $L_{5}$ of RLSp2RVSp $(T)$, and a linear combination $L_{2}$ of $T$. Suppose $L_{2}=L_{5}$. Then the support of $L_{2}=$ the support of $L_{5}$.

Proof: The support of $L_{2} \subseteq$ the support of $L_{5}$. Consider $u$ being an element of $\operatorname{RLSp} 2 \operatorname{RVSp}(T)$ such that $x=u$ and $L_{5}(u) \neq 0_{\mathbb{R}_{\mathrm{F}}}$.

(74) Let us consider a real linear space $V$, a finite sequence $F_{2}$ of elements of $V$, a function $f_{1}$ from $V$ into $\mathbb{R}$, a finite sequence $F_{4}$ of elements of $\operatorname{RLSp} 2 \operatorname{RVSp}(V)$, and a function $f_{3}$ from $\operatorname{RLSp} 2 \operatorname{RVSp}(V)$ into $\mathbb{R}_{\mathrm{F}}$. If $f_{1}=$ $f_{3}$ and $F_{2}=F_{4}$, then $f_{1} \cdot F_{2}=f_{3} \cdot F_{4}$.

(75) Let us consider a real linear space $T$, a finite sequence $F_{3}$ of elements of $T$, and a finite sequence $F_{2}$ of elements of $\operatorname{RLSp} 2 \operatorname{RVSp}(T)$. If $F_{3}=F_{2}$, then $\sum F_{3}=\sum F_{2}$.

(76) Let us consider a real linear space $T$, a linear combination $L_{5}$ of $\operatorname{RLSp2RVSp}(T)$, and a linear combination $L_{2}$ of $T$. If $L_{2}=L_{5}$, then $\sum L_{2}=\sum L_{5}$. The theorem is a consequence of (73) and (74).

Let us consider a real linear space $T$, a subset $A_{2}$ of $\operatorname{RLSp} 2 \operatorname{RVSp}(T)$, and a subset $A_{3}$ of $T$. Now we state the propositions:

(77) If $A_{2}=A_{3}$, then $\Omega_{\operatorname{Lin}\left(A_{3}\right)}=\Omega_{\operatorname{Lin}\left(A_{2}\right)}$. The theorem is a consequence of (72), (73), and (76).

(78) If $A_{2}=A_{3}$, then $A_{2}$ is linearly independent iff $A_{3}$ is linearly independent. The theorem is a consequence of (72), (73), and (76).

(79) Let us consider a real linear space $T$, a set $X$, a subspace $U$ of RLSp2RVSp $(T)$, and a subspace $W$ of $T$. Suppose $\Omega_{U}=\Omega_{W}$. Then $X$ is a linear combination of $U$ if and only if $X$ is a linear combination of $W$.

(80) Let us consider a real linear space $W$, and a set $X$. Then $X$ is a basis of $\operatorname{RLSp} 2 \operatorname{RVSp}(W)$ if and only if $X$ is a basis of $W$. The theorem is a consequence of (78) and (77). 
Let us consider a real linear space $W$. Now we state the propositions:

(81) If $W$ is finite dimensional, then $\operatorname{RLSp} 2 \operatorname{RVSp}(W)$ is finite dimensional and $\operatorname{dim}(\operatorname{RLSp} 2 \operatorname{RVSp}(W))=\operatorname{dim}(W)$. The theorem is a consequence of $(80)$.

(82) $W$ is finite dimensional if and only if $\operatorname{RLSp} 2 \operatorname{RVSp}(W)$ is finite dimensional. The theorem is a consequence of $(80)$.

(83) Let us consider a non empty natural number $n$. Then $\operatorname{RLSp} 2 \operatorname{RVSp}\left(\mathbb{R}_{\mathbb{R}}^{\operatorname{Seg} n}\right)$ $=$ the $n$-dimension vector space over $\mathbb{R}_{\mathrm{F}}$. The theorem is a consequence of (51) and (52).

(84) Let us consider real linear spaces $V, W$, and a set $X$. Then $X$ is a linear operator from $V$ into $W$ if and only if $X$ is a linear transformation from $\operatorname{RLSp} 2 \operatorname{RVSp}(V)$ to $\operatorname{RLSp} 2 \operatorname{RVSp}(W)$.

(85) Let us consider real linear spaces $X, Y$, and a linear operator $L$ from $X$ into $Y$. Suppose $L$ is bijective. Then there exists a linear operator $K$ from $Y$ into $X$ such that

(i) $K=L^{-1}$, and

(ii) $K$ is one-to-one and onto.

Proof: Reconsider $K=L^{-1}$ as a function from the carrier of $Y$ into the carrier of $X . K$ is additive. For every vector $x$ of $Y$ and for every real number $r, K(r \cdot x)=r \cdot K(x)$.

(86) Let us consider real linear spaces $X, Y, Z$, a linear operator $L$ from $X$ into $Y$, and a linear operator $K$ from $Y$ into $Z$. Then $K \cdot L$ is a linear operator from $X$ into $Z$.

Proof: Reconsider $T=K \cdot L$ as a function from $X$ into $Z$. For every elements $x, y$ of $X, T(x+y)=T(x)+T(y)$. For every real number $a$ and for every vector $x$ of $X, T(a \cdot x)=a \cdot T(x)$.

(87) Let us consider real linear spaces $V, W$, a subset $A$ of $V$, and a linear operator $T$ from $V$ into $W$. Suppose $T$ is bijective. Then $A$ is a basis of $V$ if and only if $T^{\circ} A$ is a basis of $W$. The theorem is a consequence of (84) and $(80)$.

(88) Let us consider a finite dimensional real linear space $V$, and a real linear space $W$. Suppose there exists a linear operator $T$ from $V$ into $W$ such that $T$ is bijective. Then

(i) $W$ is finite dimensional, and

(ii) $\operatorname{dim}(W)=\operatorname{dim}(V)$.

The theorem is a consequence of (87).

(89) Let us consider a finite dimensional real linear space $V$. Suppose $\operatorname{dim}(V)$ 
$\neq 0$. Then there exists a linear operator $T$ from $V$ into $\mathbb{R}_{\mathbb{R}}^{\operatorname{Seg} \operatorname{dim}(V)}$ such that $T$ is bijective. The theorem is a consequence of (81), (64), (83), and (84).

(90) Let us consider finite dimensional real linear spaces $V, W$. Suppose $\operatorname{dim}(V) \neq 0$. Then $\operatorname{dim}(V)=\operatorname{dim}(W)$ if and only if there exists a linear operator $T$ from $V$ into $W$ such that $T$ is bijective. The theorem is a consequence of (89), (85), (86), and (88).

\section{REFERENCES}

[1] Grzegorz Bancerek, Czesław Byliński, Adam Grabowski, Artur Korniłowicz, Roman Matuszewski, Adam Naumowicz, Karol Pąk, and Josef Urban. Mizar: State-of-the-art and beyond. In Manfred Kerber, Jacques Carette, Cezary Kaliszyk, Florian Rabe, and Volker Sorge, editors, Intelligent Computer Mathematics, volume 9150 of Lecture Notes in Computer Science, pages 261-279. Springer International Publishing, 2015. ISBN 978-3319-20614-1. doi 10.1007/978-3-319-20615-8_17.

[2] Grzegorz Bancerek, Czesław Byliński, Adam Grabowski, Artur Korniłowicz, Roman Matuszewski, Adam Naumowicz, and Karol Pak. The role of the Mizar Mathematical Library for interactive proof development in Mizar. Journal of Automated Reasoning, 61(1):9-32, 2018. do1:10.1007/s10817-017-9440-6

[3] Noboru Endou and Yasunari Shidama. Completeness of the real Euclidean space Formalized Mathematics, 13(4):577-580, 2005.

[4] Miyadera Isao. Functional Analysis. Riko-Gaku-Sya, 1972.

[5] Robert Milewski. Associated matrix of linear map Formalized Mathematics, 5(3):339-345, 1996.

[6] Karol Pąk. Basic properties of the rank of matrices over a field. Formalized Mathematics, 15(4):199-211, 2007. doi $10.2478 / \mathrm{v} 10037-007-0024-5$

[7] Karol Pak. Linear map of matrices. Formalized Mathematics, 16(3):269-275, 2008. doi: $10.2478 /$ v10037-008-0032-0.

[8] Laurent Schwartz. Théorie des ensembles et topologie, tome 1. Analyse. Hermann, 1997.

[9] Kôsaku Yosida. Functional Analysis. Springer, 1980.

Accepted June 30, 2021 